\title{
Association between calcium supplementation and bone mineral density in children: a systematic review and meta-analysis
}

Jin-Ping Gao ${ }^{a}$, Hong-Xia Renª, Yan-Fei Wanga, Shi-Fan Han,*, Chang-Tai Zhu ${ }^{\mathrm{b}}$

${ }^{a}$ School of Nursing, Shanxi Medical University, Taiyuan, Shanxi 030001, China

${ }^{b}$ Department of Transfusion Medicine, Shanghai Jiao Tong University Affiliated Sixth People's Hospital, Shanghai 200233, China

Received: 14 July 2020; Accepted: 17 August 2020; Published: 20 June 2021

Abstract: Objective: To evaluate the effects of calcium supplementation on bone mineral density in children.

Methods: The PubMed, Embase, and Cochrane library were systematically searched. The retrieve inception date was between October 2001 and October 2019. Two reviewers independently performed the data extraction and assessed methodology quality. Studies were limited to randomized clinical trials comparing calcium supplement with a placebo for bone mineral density in children. A meta-analysis was performed to calculate standard mean difference (SMD) and 95\% confidence interval (CI).

Results: A total of 6 randomized controlled trials involving 408 participants (calcium supplementation group: 198; placebo group: 210) were finally included in this study. The meta-analysis revealed that, compared with placebos, calcium supplementation had no effect on the bone mineral densities [the whole-body: SMD with $\mathrm{Cl}=0.43(-0.05-0.91), P=0.08, I^{2}=75 \%$; the 2nd-4th lumbar vertebrae: SMD with $95 \% \mathrm{Cl}=0.27(-0.17$ to 0.70$\left.), P=0.07, I^{2}=0 \%\right)$ ]. Sensitivity analysis revealed that the results of the whole-body bone mineral density were unstable and that the bone density of the 2nd-4th lumbar spine was robust.

Conclusions: The results of this meta-analysis suggested that calcium supplementation did not improve bone mineral density in children. However, there continues to be a need for more high-quality studies to verify this fact in the future.

Keywords: children • calcium • bone mineral density • meta-analysis • randomized controlled trial

(C) Shanxi Medical Periodical Press.

\section{Introduction}

Osteoporosis is a systemic osteopathy characterized by low bone mass, destruction of bone microstructure, increased bone brittleness, and predisposition to fractures. ${ }^{1}$ In 2011, the US National Institutes of Health proposed that osteoporosis is a disease of the bone system characterized by decreased bone strength and increased risk of fracture. Bone strength reflects 2 aspects of bone, namely, bone mineral density and bone quality. ${ }^{2}$ Osteoporosis is a

How to cite this article: Gao JP, Ren HX, Wang YF, Han SF, Zhu CT. Association between calcium supplementation and bone mineral density in children: a systematic review and meta-analysis. Front Nurs. 2021;2:169-176. 
bone disease that is closely related to age, and the prevalence rate increases with age. ${ }^{3}$ According to the latest survey data, the prevalence of osteoporosis among Chinese individuals who are $>50$ years old is $20.7 \%$ for women and $14.4 \%$ for men. The prevalence of osteoporosis is significantly increased in people $>60$ years old, especially in women. ${ }^{4}$ Osteoporosis is currently a major public health problem. Low bone density is a significant risk factor for osteoporosis. Calcium is the primary mineral that is constituent in bones; consequently, it may be hypothesized that dietary intake of Calcium contributes to the strength and hardness of bones. Calcium deposition in bones is an ongoing process from childhood to adolescence. If the optimal bone mass is not reached within this period, the impact on bone health will be irreversible. Therefore, adolescence is a very important period to prevent osteoporosis. ${ }^{5,6}$ According to data available on the national sample survey, the average calcium intake in children before 2-11 years old was less than $300 \mathrm{mg} / \mathrm{d}$, while in that of 11-18-year-old children it was the highest and less than $380 \mathrm{mg} / \mathrm{d}$, and only about $10 \%$ of the children reached the appropriate intake. ${ }^{7}$ Adequate calcium intake in childhood can not only protect the bone health of children, but also reduce the risk of osteoporosis and fracture in the future. ${ }^{8}$ If there is insufficient calcium intake during childhood, it may not be possible reach maximum peak bone mass in adulthood. Normal bone mineral density, bone mineral content, and bone mass peak value are particularly important for the growth of girls, and are very important for calcium deficiency during pregnancy and lactation. ${ }^{9-11}$ Some researchers $^{12-14}$ have conducted qualitative integration of calcium supplementation in children, and these results indicate that calcium supplementation has a favorable effect on bone outcome in children. The purpose of this study was to systematically evaluate the effects of calcium supplementation on bone development in children, and thereby provide a reference for calcium supplementation in children.

\section{Materials and methods}

\subsection{Retrieval strategy}

The review protocol was registered in the PROSPERO International prospective register of systematic reviews (CRD42020160858). A computer-based literature search was conducted in the databases PubMed, Embase, and Cochrane, using different combinations of the keywords "Calcium [Mesh]," "Calcium [Mesh]," and "Child [Mesh]" with Boolean logic symbols (AND, OR), for reports published between October 2001 and October 2019 pertaining to randomized controlled trials (RCTs). The detailed search expressions are shown in Appendix 1.

\subsection{Inclusion criteria}

Trials were selected for inclusion in the present study based on the following criteria: (1) trials comprising healthy children; (2) trials with the following intervention measures: the experimental groups were treated with calcium supplements (including food) and the control groups received placebos; (3) trials with the following outcome indicators: measurements were conducted using dual energy X-ray absorptiometry (DXA) for at least 1 year including the follow-up period, with bone mineral density (BMD) and bone mineral content (BMC) as the main outcome indicators and bone area (BA) as the secondary indicator; and (4) only RCT reports were included, with reports of high-quality quasi-trials included in the absence of suitable RCTs.

\subsection{Exclusion criteria}

Exclusion criteria were as follows: (1) non-randomized controlled trials (nRCTs); (2) trials comprising children who were diagnosed with diseases affecting bone metabolism or who were taking medications to increase bone metabolism; and (3) trials with unclear outcome indicators.

\subsection{Data extraction}

Based on the inclusion and exclusion criteria outlined above, the retrieved reports were screened by 2 independent reviewers as follows: first, after studying the titles and abstracts, the reviewers excluded duplicate reports and those that did not meet the inclusion criteria; second, the full texts of the remaining reports were studied, and the results were cross-checked; and finally, the reports for which the 2 reviewers did not reach a consensus in the first round of screening were jointly evaluated through discussion, and in the continuing absence of consensus, the reports were subjected to further evaluation by a third reviewer. The following data were extracted from the included reports: (1) general information: title, author, country, and date of publication; (2) research characteristics: general information of study subjects, including sex, age, and baseline comparability, such as the comparability of follow-up time, number of subjects followed up, management of lost follow-up data, calcium dosage, and treatment time; and (3) outcome indicators: the measurement method was DXA, the main outcome indicators were $\mathrm{BMD}$ and $\mathrm{BMC}$, and the secondary indicator was BA. 


\subsection{Data synthesis}

Statistical analysis was performed using RevMan 5.2 statistical software provided by the Cochrane Collaboration Network. Continuous outcome indicators were expressed as means \pm standard deviations, and pooled effect size was expressed as standardized mean difference (SMD). Effect variables were reported as 95\% confidence intervals (Cls) and statistical significance was tested at the level of $\alpha=0.05$; effect variables were subjected to $Q$ test and $R^{2}$ for heterogeneity. If $R^{2}<50$ (or $P>0.10$ ), the data were fitted to a fixed-effects model; otherwise, the data were fitted to a random-effects model (i.e., $P^{2}>50$ or $P<0.10$ ). Sensitivity analysis was performed to evaluate the stability of meta-analysis results, or alternatively, meta-analysis results were recalculated using a different effects model and effect size (i.e., interchange of mean difference (MD) and SMD). Meta-analysis results are considered stable if they did not change after the interchange; otherwise, they were considered unstable, and caution should be exhibited in the interpretation of the results.

\section{Results}

\subsection{Literature retrieval}

A total of 387 reports were retrieved, of which 42 were from PubMed, 239 from Cochrane, and 107 from Embase. After the exclusion of 14 duplicate reports, 373 remained for further screening, which led to the exclusion of 241 reports based on their titles and abstracts, thus leaving 132 reports as the remainder. Of these, 101 were excluded after study of their full texts, leaving 31 reports on RCTs involving calcium. Of these, 15 involved nonconforming intervention measures, 4 included nonconforming subjects, 4 reported nonconforming outcome indicators, 1 did not include a placebo group, and 1 was based on a different type of trial. After exclusion of these 25 reports, $4 \mathrm{RCTs}{ }^{15-18}$ were finally included in this study, comprising a total of 408 participants, of which 198 were treated with calcium supplements and 210 were given placebos (Figure 1). The selected research sources were the UK, Australia, and India. Basic information about the literature is shown in Table 1.

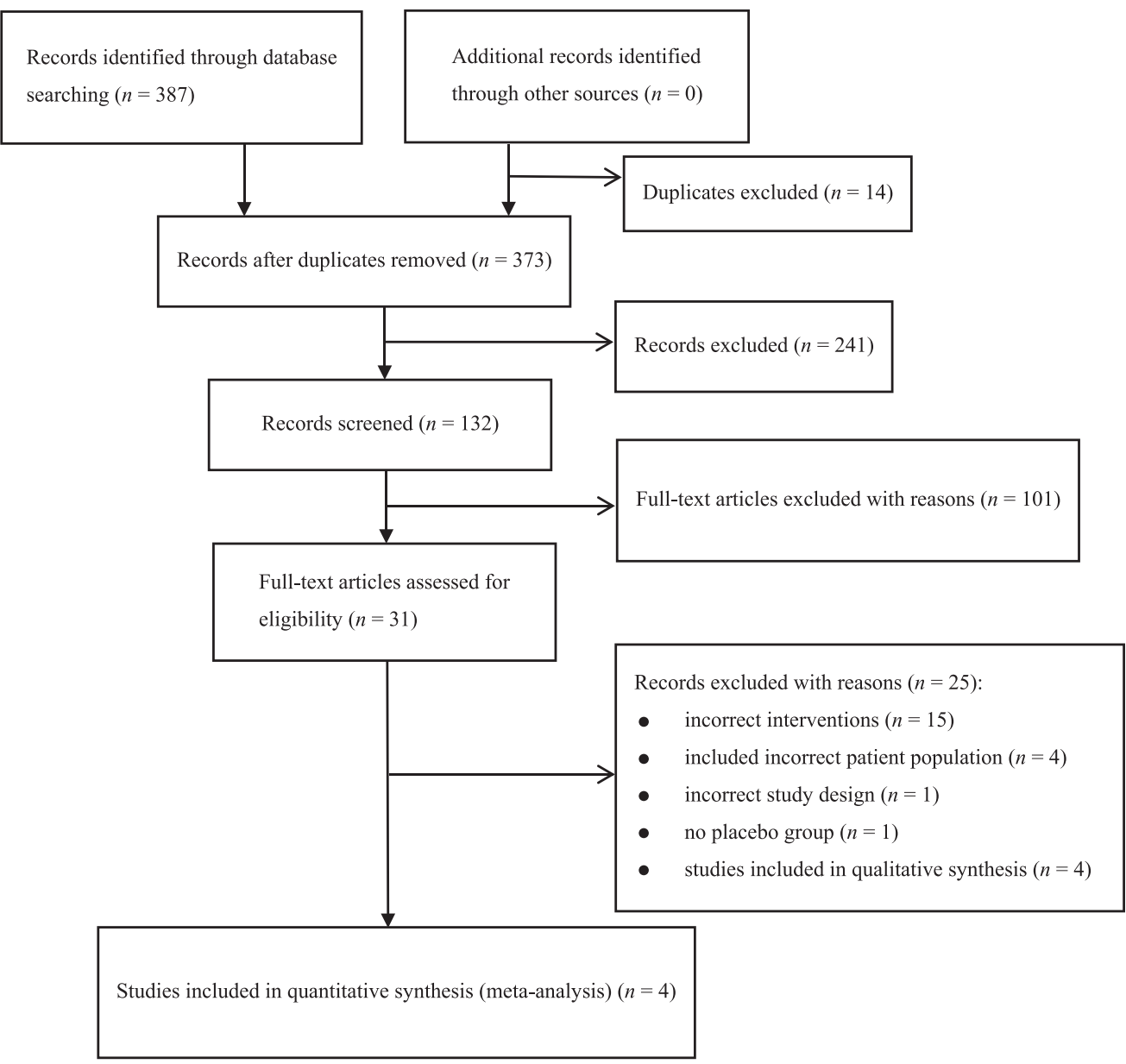

Figure 1. Literature screening process. 


\subsection{Risk of bias in the included RCTs}

The included RCTs were comparable with respect to the general characteristics at baseline (Table 1). The risk of bias in the included RCTs was evaluated using a bias risk assessment tool of the Cochrane Collaboration Network. Of the four included RCTs, only one ${ }^{18}$ elaborated on the randomization method, while the other three ${ }^{15-17}$ described their randomization methods only in a narrative manner with many details missing, and only one ${ }^{16}$ described allocation concealment methods. With the exception of one study, ${ }^{15}$ the researchers and subjects were blinded in all the other studies; only one ${ }^{15}$ was blind for outcome evaluators; four were described to be withdrawn and lost to follow-up data, and intentionto-treat analysis was performed in the RCT reporting lost to follow-up data (Figure 2).

\subsection{Meta-analysis of the whole-body BMD}

Three RCTs ${ }^{15,16,18}$ reported on the whole-body BMD. Heterogeneity test results showed that there was heterogeneity among studies of whole-body BMD $\left(I^{2}=75 \%\right.$, $P=0.02)$; the random-effect model was used to combine the effects. The meta-analysis adopted the random effect model. The results of meta-analysis showed that calcium supplementation had no effect on the whole-body BMD (SMD $=0.43,95 \% \mathrm{Cl}=-0.05,0.91$, $P=0.08)$ (Figure 3).

\subsection{Meta-analysis of the 2 nd-4th lumbar spine BMD}

Two RCTs ${ }^{15,17}$ reported on the 2 nd-4th lumbar vertebrae BMD. Heterogeneity test results showed that there was homogeneity among studies of the 2nd-4th lumbar spine $\operatorname{BMD}\left(I^{2}=0 \%, P=0.85\right)$; the random-effects model was used to combine effects. The results showed that calcium supplementation had no effect on the $2 \mathrm{nd}-$ 4th lumbar spine BMD (SMD $=0.30,95 \% \mathrm{Cl}=-0.02$, $0.61, P=0.07$ ) (Figure 4).

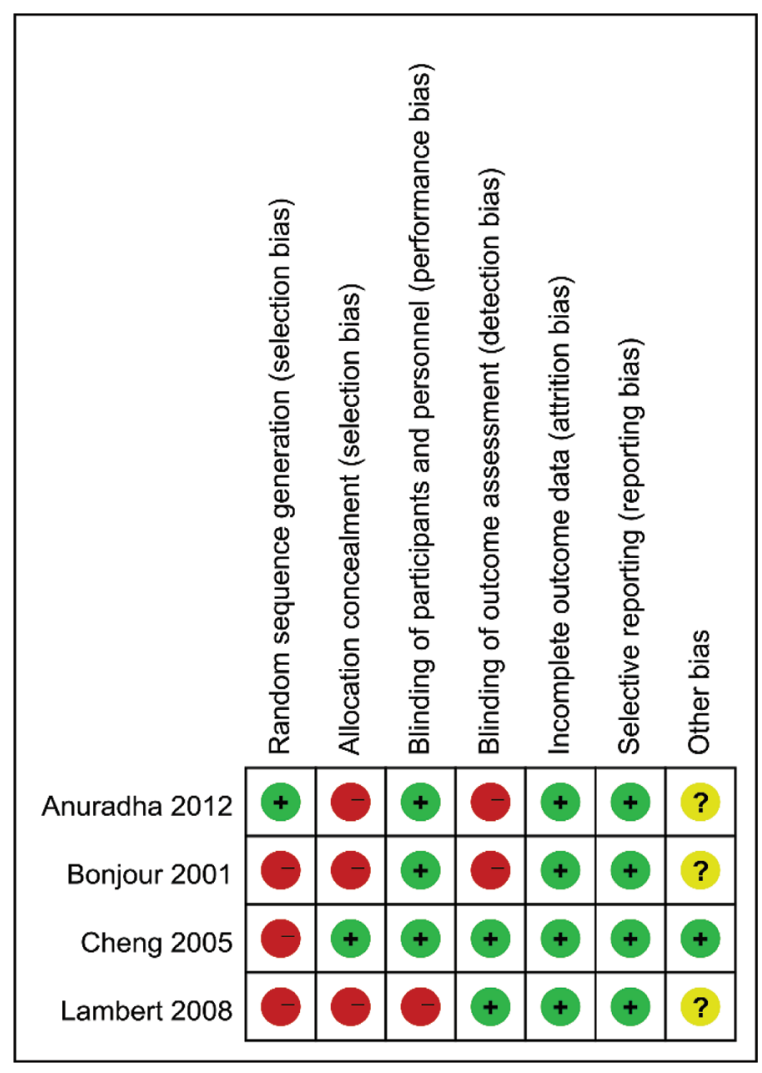

Figure 2. Risk bias evaluation diagram.

\begin{tabular}{|c|c|c|c|c|c|c|c|c|c|c|}
\hline \multirow{2}{*}{$\begin{array}{l}\text { Inclusive } \\
\text { RCTs }\end{array}$} & \multirow[b]{2}{*}{ Population } & \multicolumn{2}{|c|}{ Sample size } & \multirow{2}{*}{$\begin{array}{l}\text { Ages } \\
\text { (years) }\end{array}$} & \multicolumn{2}{|c|}{ Intervention measures } & \multirow{2}{*}{$\begin{array}{l}\text { Intervention } \\
\text { duration } \\
\text { (years) }\end{array}$} & \multirow[b]{2}{*}{ Dose (mg) } & \multirow{2}{*}{$\begin{array}{l}\text { Percentage } \\
\text { of lost visits } \\
(\%)\end{array}$} & \multirow{2}{*}{$\begin{array}{l}\text { Outcome } \\
\text { indicators }\end{array}$} \\
\hline & & $\begin{array}{l}\text { Control } \\
\text { group }\end{array}$ & $\begin{array}{l}\text { Intervention } \\
\text { group }\end{array}$ & & I & C & & & & \\
\hline $\begin{array}{l}\text { Cheng } \\
2005^{12}\end{array}$ & $\begin{array}{l}\text { Healthy } \\
\text { children }\end{array}$ & 41 & 39 & $10-12$ & Calcium & Placebo & 2 & 1000 & 18.7 & $\begin{array}{l}\text { Whole-body } \\
\text { BMD }\end{array}$ \\
\hline $\begin{array}{l}\text { Anuradha } \\
2012^{14}\end{array}$ & $\begin{array}{l}\text { Healthy } \\
\text { children }\end{array}$ & 70 & 69 & $8-12$ & Calcium & $\begin{array}{l}\text { Multivitamin } \\
\text { tablets }\end{array}$ & 1 & 500 & 1.8 & $\begin{array}{l}\text { Whole-body } \\
\text { BMD }\end{array}$ \\
\hline $\begin{array}{l}\text { Lambert } \\
2008^{10}\end{array}$ & $\begin{array}{l}\text { Healthy } \\
\text { children }\end{array}$ & 37 & 36 & $11-12$ & $\begin{array}{l}\text { Calcium } \\
\text { fortified } \\
\text { fruit drinks }\end{array}$ & $\begin{array}{l}\text { Regular fruit } \\
\text { drinks }\end{array}$ & 1 & 792 & 7.2 & $\begin{array}{l}\text { Whole-body and } \\
\text { 2nd-4th lumbar } \\
\text { vertebrae BMD }\end{array}$ \\
\hline $\begin{array}{l}\text { Bonjour } \\
2001^{13}\end{array}$ & $\begin{array}{l}\text { Healthy } \\
\text { children }\end{array}$ & 62 & 54 & $7-9.3$ & Calcium & Placebo & $3-5$ & 850 & 24.1 & $\begin{array}{l}\text { 2nd-4th lumbar } \\
\text { vertebrae BMD }\end{array}$ \\
\hline
\end{tabular}

Note: I, intervention group; C, control group; BMD, bone mineral density.

Table 1. Basic characteristics of included RCTs. 


\subsection{Sensitivity analysis}

The sensitivity analysis showed that the results of the whole-body BMD change significantly after the transition from the random-effects model to the fixedeffects model and exclusion of articles one by one (Figures 5 and 6), and the 2nd-4th lumbar vertebrae BMD did not change significantly after changing the fixed-effects model to the random-effects model and exclusion of articles one by one (Figures 7 and 8 ).

\subsection{Publication bias}

Because only 4 RCTs were included, no analysis of publication bias was performed in this study.

\section{Discussion}

Childhood and adolescence are periods of the most significant bone development. BMD can directly reflect bone development, and it has a significant impact on the prevention of osteoporosis and fractures. ${ }^{19,20}$ In this study, the results of the relevant RCT studies were summarized. Comparison between the trial groups (who were treated with calcium supplements) and control groups (who were receiving placebos) showed that routine calcium supplementation had no effect on the whole body and 2nd-4th lumbar vertebrae BMD. Winzenberg et al. $^{6}$ also showed that there was no effect of calcium supplementation on the femoral neck or lumbar spine BMD.

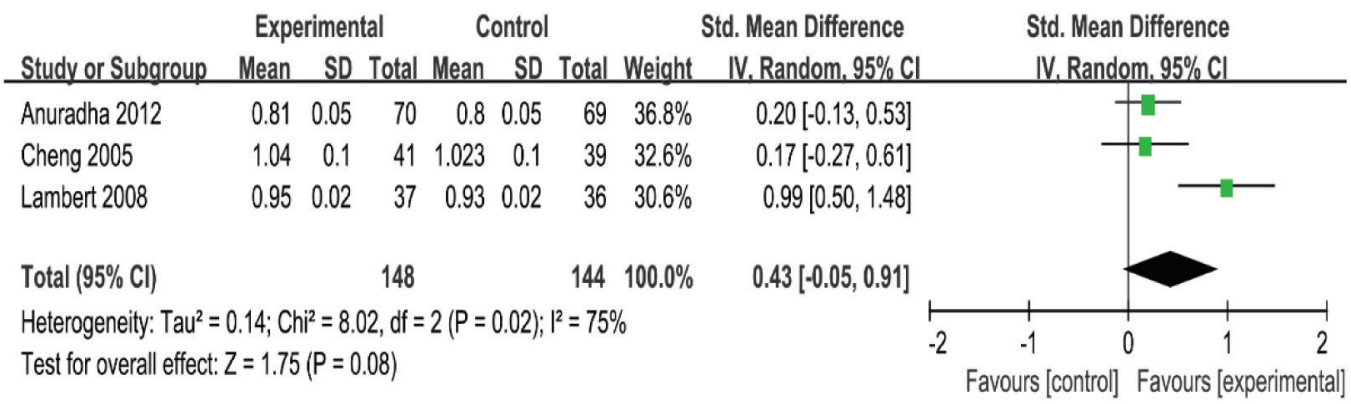

Figure 3. Meta-analysis of the effects of calcium supplements and placebo on the whole-body bone mineral density in healthy children

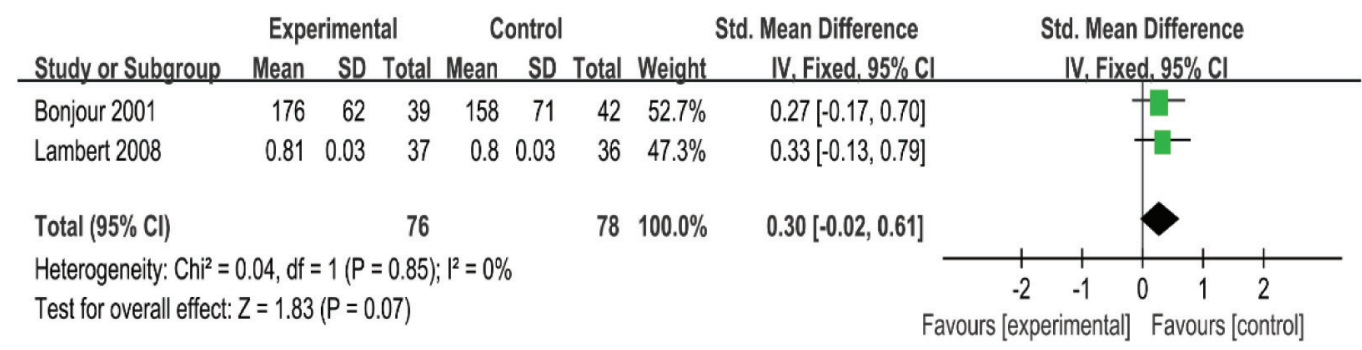

Figure 4. Meta-analysis of the effects of calcium supplements and placebo on the 2nd-4th lumbar vertebrae bone mineral density in healthy children.

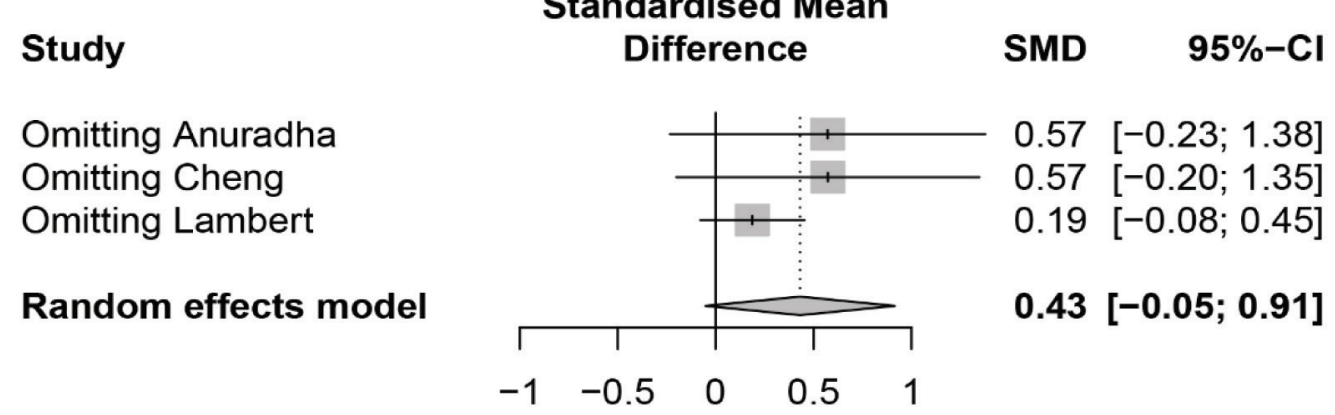

Figure 5. Meta-analysis of the effects of calcium supplement and placebo on the whole-body BMD in healthy children after exclusion of articles one by one. 


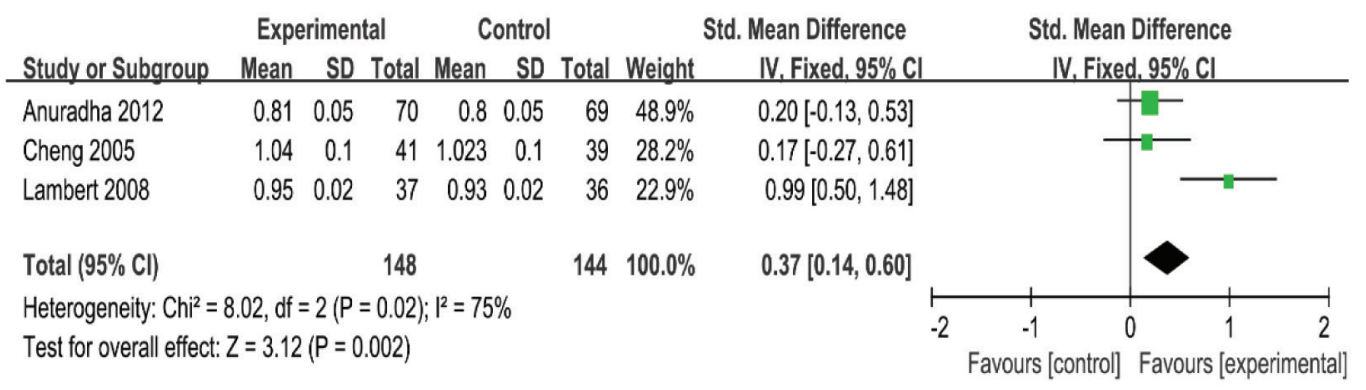

Figure 6. Meta-analysis of the effects of calcium supplements and placebo by fixed-effects model on the whole-body BMD in healthy children.

Study

Omitting Bonjour
Omitting Lambert

Fixed effect model

\section{Standardised Mean Difference}

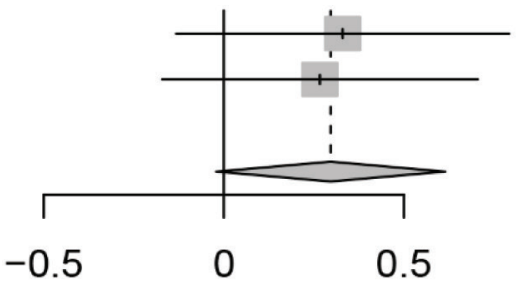

SMD $\quad 95 \%-\mathrm{Cl}$

$0.33[-0.13 ; 0.79]$

$0.27[-0.17 ; 0.70]$

$0.30[-0.02 ; 0.61]$

Figure 7. Meta-analysis of the effects of calcium supplement and placebo on the 2nd-4th lumbar vertebrae BMD in healthy children after exclusion of articles one by one.

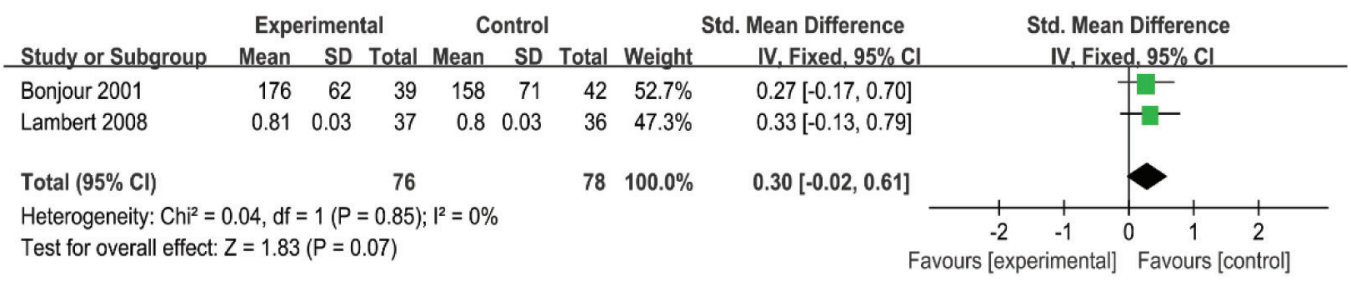

Figure 8. Meta-analysis of the effects of calcium supplements and placebo by random-effects model on the 2nd-4th lumbar vertebrae BMD in healthy children.

The timing and dosage of calcium supplementation are of great importance. Given that bone growth is different at different developmental stages, calcium requirements differ as well. In a study by Ma et al. ${ }^{21}$ trial groups were divided into a high-calcium subgroup, a medium-calcium subgroup, and a lowcalcium subgroup, and increasing calcium intake was observed to promote increases in bone mass in adolescents. Although this effect was moderate, an increase in BMC of the femoral neck might reduce the risk of osteoporosis and fractures. Calcium supplementation in early adolescence had a more significant effect than supplementation at later stages. Due to the small number of studies included in this study, the time and dosing of calcium supplementation were not analyzed.

Calcium intake contributes to bone formation, and the role of calcium in bone consolidation is gradually reduced. ${ }^{22,23}$ Since calcium supplementation reduces bone remodeling, rather than increasing it, some studies have observed short-term benefits. If calcium for bone reconstruction effect is greater than the effect on bone modeling, in such a way that the duration of calcium for the bone growth differences becomes very small, it is said that as the calcium time growth takes place, bone mineral density/bone mineral content will decline; however, this statement has not been confirmed, since there is a need for more RCTs to verify it. 
The sensitivity analysis of the whole-body bone density indicates that this conclusion is not adequately stable and that the results need to be treated with caution. This may be due to the small amount of literature included, and more high-quality studies must be included in the future to further verify this result.

Limitations of this study include the following aspects: first, due to the limitation of the quantity and quality of the included studies, the conclusions of this study still need to be verified by more high-quality RCTs. Second, there no study could be retrieved on calcium dosage and children's age; for this reason, it was impossible to obtain data which presents information on the correlation prevailing between the optimal pediatric dosage of calcium and age of children.

\section{References}

1. Liu ZH. Osteoporosis. Beijing: Science Press; 2001 (in Chinese).

2. NIH Consensus Development Panel on Osteoporosis Prevention, Diagnosis, and Therapy, March 7-29, 2000: highlights of the conference. South Med J. 2001;94:569-573.

3. Chinese Society of osteoporosis and Bone Mineral Research (CSOBMR). Guidelines for diagnosis and treatment of primary osteoporosis. Chin J Osteo Bone Miner Res. 2011;4:2-17 (in Chinese).

4. People's Daily Online. The Health Commission releases the results of the first osteoporosis epidemiological survey in China. Web site. http://k.sina. com.cn/article_2194948720_82d4427002000d5cy. html. Accessed November 7, 2019.

5. Ma BL, Cai MQ. Influencing factors of calcium requirement and osteoporosis. Chin $J$ Clin Nutr. 2006;14:265-268 (in Chinese).

6. Winzenberg TM, Shaw K, Fryer J, Jones G. Calcium supplementation for improving bone mineral density in children. Cochrane Database Syst Rev. 2006;2:CD005119.

7. Qin R. Consensus among Chinese experts on calcium nutrition for children (2019 edition). Chin J Woman Child Health Res. 2019;30:262-269 (in Chinese).

8. Jiang F. Effects of calcium nutrition and exercise on adult bone health in children and adolescents. Chin J Pract Pediatr. 2012;27:174-178 (in Chinese).

9. Wu KM. Children vitamin D, calcium nutrition reasonable supplement. Chin J Pract Pediatr. 2012;27:165-169 (in Chinese).

10. Zhu QJ. Effects of calcium supplementation and milk drinking on growth and bone mineral density

\section{Conclusions}

Existing evidence suggests that calcium supplementation did not improve BMD in children. Therefore, calcium supplements are discouraged as a routine form of prevention in healthy children.

\section{Ethical approval}

Ethical issues are not involved in this paper.

\section{Conflicts of interest}

All contributing authors declare that no conflicts of interest exist.

in children. Women's Health Res. 2016;9:11-12 (in Chinese).

11. Meng QT, Diao QP, Zhang YX, Hou DY. The harm of calcium deficiency in preschoolers and the method of calcium supplement are analyzed. J Anshan Normal Univ. 2013;15:62-66 (in Chinese).

12. Prentice A, Ginty F, Stear SJ, Jones SC, Laskey MA, Cole TJ. Calcium supplementation increases stature and bone mineral mass of 16- to 18-year-old boys. J Clin Endocrinol Metab. 2005;90:3153-3161.

13. Lanou AJ, Berkow SE, Barnard ND. Calcium, dairy products, and bone health in children and young adults: a reevaluation of the evidence. Pediatrics. 2005;115:736-743.

14. WosjeKS, Specker BL. Role of calcium in bone health during childhood. Nutr Rev. 2000;58:253-268.

15. Lambert HL, Eastell R, Karnik K, Russell JM, Barker ME. Calcium supplementation and bone mineral accretion in adolescent girls: an 18-mo randomized controlled trial with 2-y follow-up. Am J Clin Nutr. 2008;87:455-462.

16. Cheng $S$, Lyytikäinen $A$, Kröger $H$, et al. Effects of calcium, dairy product, and vitamin D supplementation on bone mass accrual and body composition in 10-12-y-old girls: a 2-y randomized trial. Am J Clin Nutr. 2005;82:1115-1148.

17. Bonjour JP, Chevalley T, Ammann P, Slosman D, Rizzoli R. Gain in bone mineral mass in prepubertal girls 3.5 years after discontinuation of calcium supplementation: a follow-up study. Lancet. 2001;358:1208-1212.

18. Khadilkar A, Kadam N, Chiplonkar S, Fischer PR, Khadilkar V. School-based calcium-vitamin D with micronutrient supplementation enhances bone 
mass in underprivileged Indian premenarchal girls. Bone. 2012;51:1-7.

19. Fang $H Y$, Gao $H Q$. The main factors affecting the bone density level of children were studied. Gansu Med J. 2015;34:844-845 (in Chinese).

20. Yan JH, Wang Y. Research progress on factors influencing bone mineral density in children. J Sichuan North Med Sch. 2017;32:147-151 (in Chinese).

21. Ma XM, Huang ZW, Yang XG, Su YX. Calcium supplementation and bone mineral accretion in Chinese adolescents aged 12-14 years: a 12-month, dose-response, randomised intervention trial. $\mathrm{Br} \mathrm{J}$ Nutr. 2014;112:1510-1520.

22. Matkovic V, Goel PK, Badenhop-Stevens NE, et al. Calcium supplementation and bone mineral density in females from childhood to young adulthood: a randomized controlled trial. Am J Clin Nutr. 2005;81:175-188.

23. Lee WT, Leung SS, Leung DM, Cheng JC. A followup study on the effects of calcium-supplement withdrawal and puberty on bone acquisition of children. Am J Clin Nutr. 1996;64:71-77. 